\title{
Bioeconomía y derechos humanos en América Latina*
}

\author{
Bioeconomics and human rights in Latin America
}

\section{Bioeconomia e direitos humanos na América Latina}

\author{
Adriana Alejandra Gallego-Marín \\ Estudiante de Derecho Universidad Libre Seccional Cali. \\ alega0302@gmail.com
}

\section{María Alejandra Ramírez-Galvis}

Abogada. Defensora de Derechos Humanos. Investigadora Universidad Santo Tomás, Bogotá - Colombia. maralejramirez@gmail.com

\section{Andrés David Arana-Gutiérrez}

Estudioso de las relaciones de poder del capital corporativo global y de los movimientos sociales, populares y comunitarios en América Latina. Estudiante de Derecho Universidad Libre Seccional Cali andresarana2012@hotmail.com

\section{Orfa Margarita Giraldo-Alzate}

Abogada, Magíster en Filosofía. Universidad de Baja California, México orfa.giraldo@unilibrecali.edulco

\section{Martha Isabel Cabrera-Otálora}

Licenciada en Filosofía, Especialista en Pedagogía para el Desarrollo del Aprendizaje Autónomo, Magíster en Educación. Docente Investigadora Universidad Nacional Abierta y a Distancia UNAD, Palmira, Colombia. martha.cabrera@unad.edu.co

\section{Libia Esperanza Nieto-Gómez}

Ingeniera Agrícola, Especialista en Recursos Hidráulicos Universidad Nacional de Colombia. Docente Investigadora Universidad Nacional Abierta y a Distancia UNAD, Bogotá, Colombia. libia.nieto@unad.edu.co

\section{Reinaldo Giraldo-Díaz}

Doctor en Filosofía, Universidad de Antioquia, Colombia. Magíster en Filosofía de la Universidad del Valle, Colombia. Ingeniero Agrónomo de la Universidad Nacional de Colombia. Grupo de Investigación "Ignacio Torres Giraldo". Docente Asistente - Universidad Nacional Abierta y a Distancia - UNAD - Palmira, Colombia. reinaldo.giraldo@unad.edu.co

\section{Resumen}

A partir de las experiencias de violación sistemática y generalizada de los Derechos Humanos en América Latina, promovida por las políticas extractivistas de las corporaciones

* Cómo citar: Gallego-Marín, A. A., et al. (2016). Bioeconomía y derechos humanos en América Latina. Revista Libre Empresa, 13(2), 131 141 http://dx.doi.org/10.18041/libemp.2016.v13n2.26209 
multinacionales y los Estados, se analizan las condiciones de posibilidad para la agudización de esta violación dados los roles que son exigidos en el nuevo orden mundial para el desarrollo del mercado bioeconómico. Se encontró que existe un ordenamiento jurídico supranacional consistente en que acuerdos, convenios y tratados internacionales tienden cada vez más a brindar garantías para la inversión y los inversionistas, que a proteger los derechos y la vida de las comunidades, sus territorios y recursos ambientales. Se concluye que los pueblos de América Latina para protegerse de la agresión de los Estados y de las corporaciones multinacionales deben fortalecer sus procesos organizativos y, en el ámbito supranacional, acudir a los tribunales ético políticos. Es necesario propender por una bioeconomía orientada hacia la agricultura sostenible en la que se tengan en cuenta las necesidades de las comunidades y una activa participación de éstas y de los investigadores conscientes de que es posible lograr un equilibrio entre estos factores.

\section{Palabras clave}

Estado social de derecho, Estado de rectoría, resistencia.

\section{Abstract}

Based on the experiences of systematic and widespread violations of human rights in Latin America promoted by the extractivist policies of multinational corporations and states, the conditions of possibility for the exacerbation of this violation are analyzed given the roles that are demanded in The new world order for the development of the bioeconomic market. It was found that there is a supranational legal order in which international agreements, agreements and treaties increasingly tend to provide guarantees for investment and investors, to protect the rights and life of communities, their territories and environmental resources. It is concluded that the peoples of Latin America to protect themselves from the aggression of States and multinational corporations must strengthen their organizational processes and, in the supranational sphere, turn to the ethical political tribunals. There is a need for a sustainable agriculture-oriented bioeconomy that takes into account the needs of the communities and actively participates in them and of the researchers who are aware that it is possible to achieve a balance between these factors.

\section{Key words}

Social state of law, State of rectory, resistance.

\section{Resumo}

A partir das experiências de raiz sistemática e gerada pelos direitos humanos em América Latina, promovida pelas políticas extractivistas das corporações multinacionais e pelos estados, analisada as condições de oportunidade para a agudização de estações, O novo orden mundial para o desenvolvimento do mercado bioeconômico. Se encontró que existe um ordenamento jurídico supranacional consistente em que acordos, convenções e tratados internacionais são cada vez mais um brindar garantias para a inversão e os inversionistas, que a proteger os direitos ea vida das comunidades, seus territórios e recursos ambientais. Se concluye que os pueblos de América Latina para protegido da agência dos Estados e das corporações multinacionais se fortalecem os processos organizativos e no âmbito 
supranacional, acudir aos tribunais políticos éticos. Es necessario propender por una bioeconomìa orientada hacia la agricultura sustentable en la que se tiene en cuenta las necesidades de las comunidades y una activa participación éstas y de los investigadores conscientes de que es posible lograr un equilibrio entre estos factores.

\section{Palavras chave}

Estado social de direito, Estado de rectoría, resistencia.

\section{Introducción}

La OCDE (Organización para la Cooperación del Desarrollo Económico) fundamenta la configuración del nuevo orden bioeconómico al año 2030. Los países de la OCDE alinean sus políticas de desarrollo, sus políticas sociales y económicas, con el fin de fortalecer las demandas del creciente mercado bioeconómico (OECD, 2009). En este contexto, los países de la periferia, llamados tercermundistas, en vías de desarrollo, juegan un papel marginal en su participación en los dividendos que se generan como producto de las transacciones comerciales. Sin embargo, tienen un papel protagónico a la hora de asumir los costes sociales, ambientales y culturales.

En los distintos ámbitos de las actividades sociales se desarrollan productos y servicios basados en el valor que adicionan las ciencias biológicas, en especial, la biotecnología aplicada a la producción primaria, a la salud y a la industria. La incursión de estos productos y servicios de la vida cotidiana modifica las formas de vida a favor de un régimen de producción que busca la generación de plusvalía.

La agricultura se concibe con aplicación de biotecnología para mejorar variedades de plantas y animales, lo cual requiere ampliación del número de empresas e institutos de investigación que pueden utilizar la biotecnología (en particular en los países en desarrollo). En materia de salud, se formula el desarrollo de sistemas de regulación, investigación, y de historiales médicos que puedan vincular historias médicas, prescripción, información genética y otros, para apoyar la investigación y seguimiento a largo plazo de los resultados. En la industria, se pretende aumentar el apoyo a la adopción y uso de las normas internacionalmente aceptadas para el análisis del ciclo de vida, junto con otros incentivos para recompensar a tecnologías ambientalmente sostenibles, por ejemplo, impulsar la investigación en biocombustibles de alta densidad de energía (OECD, 2009; Giraldo \& Nieto, 2015).

Como consecuencia lógica de la adopción de los desarrollos de las ciencias biológicas para fortalecer el mercado mundial, se generan unos roles en los que se siguen agudizando las distinciones entre países desarrollados y subdesarrollados (denominados también países del centro y países de la periferia); mientras estos últimos son vistos como proveedores de materias primas de la tecnociencia, aquellos desarrollan y aprovechan las tecnologías, productos y servicios generados para el creciente mercado.

Ante la dinámica mundial que deviene en participación mercantil entre los países, se ha ido fortaleciendo no sólo el marco jurídico, mediador de los procesos comerciales, sino que 
se materializan políticas supranacionales que formalizan los términos de interacción entre los Estados y sus transacciones. Acuerdos, convenios y tratados internacionales tienden cada vez más a brindar garantías para la inversión y los inversionistas, que a proteger los derechos y la vida de las comunidades (sus territorios y sus recursos ambientales), lo que genera una dinámica de disputa por los territorios entre las comunidades y las empresas con las cuales tienen intereses económicos en estos.

\section{Desarrollo}

\section{Bioeconomía y Estado de rectoría en América Latina}

La configuración bioeconómica mundial se ve propiciada por la implantación de una política de seguridad jurídica que beneficia las nuevas dinámicas de acumulación. Dávalos (2011) ofrece una lectura al respecto, refiriéndose a la relación entre el Estado de seguridad jurídica y los derechos humanos. Según este autor, el Estado social de derecho ha sido erigido por el proyecto neoliberal para América Latina. Para sustentar su postura, analiza cómo se llegó al Estado social de derecho, por qué su conformación obtuvo el consenso social, cuál fue el proceso de su constitución formal y factual y qué implicaciones tiene la noción del Estado social de derecho dentro del proyecto neoliberal. Bajo estas cuatro preguntas el intelectual explicita dos hipótesis; la primera hace referencia al denominado "estado de rectoría", el cual se asume como la conclusión de los procesos de reforma política del Estado y la descentralización que llevaron a cabo el Banco Mundial y el complejo institucional de la reforma estructural. Esto es, una garantía que el Estado de rectoría establece a los individuos sobre la base de la elaboración de las políticas públicas; entre ellos los derechos de propiedad. Esta forma se caracteriza, en otras palabras, como todo espacio libre en manos del mercado bajo la sanción del Estado Social de Derecho, de manera que la política pública intervenga el Estado y la sociedad. En segundo lugar, hace alusión al "Estado de seguridad jurídica" que nace de la reforma estructural del Banco Mundial y sus proyectos sectoriales.

Ahora bien, estos roles "nuevos" que asignan las corporaciones transnacionales a los Estados, generan actualizaciones en los sistemas jurídicos de las naciones, en favor de los requerimientos de la "inversión extranjera directa". Los derechos humanos, la dignidad humana, aunque siempre han sido formalidades en los discursos de los Estados, en Colombia, son relegados aún más. No importa si un Estado como el colombiano, que históricamente ha violentado los derechos humanos y la dignidad humana (la cifra oficial de desplazamiento forzado bastaría para sustentar esta afirmación, sin embargo, cabe mencionar las desapariciones forzosas, los asesinatos extrajudiciales, las masacres a los pobladores rurales), agudiza su crisis humanitaria, sino si su seguridad jurídica está en conveniencia con la inversión extranjera directa que tendrá. Así, pues, un país obtendrá la respetable calificación por parte del foro de Davos como país altamente competitivo y favorable para la inversión extranjera directa o la consideración de "Estado fallido", excluido del crecimiento económico y condenado a la pobreza (Dávalos, 2011: 276).

Los desplazados internos constituyen el mayor colectivo de víctimas registradas: 6.897.450. En 2015 se registraron 76.017 nuevos desplazados. Este enorme desafío de 
atención y reparación requiere acción en zonas apartadas de recurrente desplazamiento, como la Costa Pacífica y Antioquia, y protección especial para las comunidades indígenas y afrocolombianas (Alto Comisionado de las Naciones Unidas para los Derechos Humanos, 2016, p.13).

En materia de desaparecidos, la Fiscalía registra más de 70.000; el Registro Nacional de Desaparecidos 19.855 hombres y 2.511 mujeres; la Unidad para la Atención y Reparación Integral a las Víctimas (UARIV) 45.515. El Registro Nacional de Desaparecidos registró 5.482 desapariciones entre enero y noviembre de 2015, de las cuales 105, incluyendo 18 mujeres, son presuntamente forzadas. En octubre, la Comisión Nacional de Búsqueda inició la revisión de sus registros con las entidades intervinientes y las organizaciones sociales a fin de contar con información más confiable. (Alto Comisionado de las Naciones Unidas para los Derechos Humanos, 2016, p. 4)

Según cifras oficiales del Grupo de Memoria Histórica se reportaron desde 1978 a 2012, 1982 masacres con un saldo de 11,751 víctimas de las que 158 fueron ejecutadas por la Fuerza Pública (Centro Nacional de Memoria Histórica, s.f.).

Hasta junio de 2014, la Fiscalía se encontraba investigando más de 3.500 casos de Ejecuciones ilegales cometidos por 785 miembros del Ejército (Nacional Human Rights Watch, 2015).

El 2015 dejó una cifra de 682 casos de agresiones a Defensores de DDHH, con 539 amenazas y 63 homicidios (Comisión Interamericana de Derechos Humanos 2011) la Defensoría del pueblo reportó que durante el mismo periodo, 472 líderes sindicales, 628 defensores de derechos humanos y 131 periodistas recibieron amenazas ( Publimetro, 2016); además el informe anual de "Front Line Defenders" estableció que entre enero y noviembre de 2015 en América Latina fueron asesinados más de 87 defensores de derechos humanos (DDHH), 54 de los cuales eran colombianos (Peace Brigades International-PBI 2016).

Quede claro que "seguridad jurídica" no es sinónimo de seguridad ciudadana. El Estado social de derecho, en Latinoamérica, ha sido relacionado en ocasiones con violencia concentrada y organizada de las corporaciones multinacionales contra las comunidades, contra los pueblos de Abya Yala. El Estado se ve así forzado a contener la resistencia del pueblo, de la sociedad civil, de las comunidades en favor de las políticas de acumulación del Banco Mundial, del Fondo Monetario Internacional, de la Organización Mundial del Comercio, de las Naciones Unidas...

El discurso de los derechos humanos, de la dignidad humana en Latinoamérica, no es abandonado por los Estados. Es aprovechado, en muchas ocasiones, para la criminalización social, para afianzar la acumulación ampliada de capital. Las resistencias sociales son criminalizadas, asimiladas por el Estado como terrorismos e inscritas en la geometría variable de la lucha mundial contra el terrorismo. Ya Atilio Borón (2007) deja claro este asunto al representar a dos tipos de terrorismos, uno que es causado por las corporaciones 
multinacionales en contubernio con los Estados y otro que está referido a las resistencias frente a la lógica y las dinámicas del capital corporativo transnacional.

Molano (2016) permite ver cómo operan de modo nefasto para las comunidades en Colombia este tipo de mangualas entre el Estado y las corporaciones multinacionales. Con respecto al Tratado de Libre Comercio (TLC) entre Canadá y Colombia, Molano señala que mientras el discurso oficial del gobierno y los Estados es de éxito y mutuo elogio, lo que se nota es que a medida que se aumentan las ganancias (recuperación de las inversiones y aumento del capital para los inversionistas) hay una mayor violación, sistemática y generalizada, de los derechos humanos en Colombia.

El panorama de los derechos humanos en las zonas donde Canadá invierte, "Hablo en primer lugar de las zonas mineras y sobre todo antes de que se haya invertido un solo peso. Allí han entrado a saco los paramilitares, amparados por la fuerza pública, como en los Llanos Orientales. Antes de que las empresas petroleras entraran el primer taladro —aunque después de que sus técnicos habían detectado los bolsones de petróleo-, entraron Martín Llanos y Cuchillo, con sus hombres y sus motosierras. La masacre de Mapiripán sembró el terror y abrió las puertas a la seguridad inversionista. Los casos se pueden multiplicar. La limpieza social precede a las masacres y las masacres a las inversiones en todas las zonas donde se prevén grandes proyectos de explotación petrolera, minera, palmera, azucarera, ganadera” (Molano, 2016).

Aunque formalmente los dos países firman un acuerdo de cooperación laboral y otro de evaluación de los derechos humanos, "Nada dicen, por ejemplo, de los atropellos de la Pacific Rubiales en Puerto Gaitán; de las amenazas a los mineros tradicionales de Marmato; de la represión de las protestas en La Colosa, en Caramanta, en Santurbán o en el Ariari. En la gran mayoría del medio centenar de empresas canadienses que hay en Colombia no ha sido posible la creación de sindicatos, sólo en dos” (Molano, 2016).

La finalidad es notoria en este contexto cuando se trata de salvaguardar y garantizar los derechos (de propiedad, de los inversionistas y sus inversiones) en un Estado que resuelve conflictos persiguiendo y reprimiendo las luchas sociales. El Estado representa y defiende el capital corporativo transnacional y privatizador. Por tanto, las disputas por la soberanía de las comunidades, en los distintos territorios del país, siempre se resuelven por el Estado a favor de las corporaciones multinacionales, dado que las adecuaciones jurídicas han sido realizadas en procura de este objetivo. De igual manera ocurre cuando estas demandas superan el ámbito del Estado y se acude al ordenamiento jurídico internacional, llegando a constituirse éste en obstáculo para las demandas de los pueblos. Cuando alguna instancia supranacional "resuelve a favor" de las comunidades, se ha observado una situación que las enmarca en una condición de pérdida por las condiciones a las que se han visto sometidas sin recibir una contraprestación que signifique reparación integral.

Según fuentes oficiales del gobierno canadiense, más del 75\% de las empresas mineras en el mundo están registradas en Canadá. Con leyes, una fiscalidad y una política extranjera favorables a las empresas extractivas, Canadá ofrece un contexto floreciente 
para la industria minera. Por tanto, los megaproyectos extractivos son hoy una causa mayor de atentados a los derechos de las colectividades. En América latina, existen en la actualidad cerca de 200 conflictos sociales oponiendo las comunidades, compañías mineras y gobiernos sobre la cuestión minera. Para las personas y colectividades que sufren la violación de sus derechos por las actividades mineras, existen numerosas barreras en el acceso a la justicia (Tribunal Permanente de los Pueblos, s. f.).

En Latinoamérica no son pocos los casos en los que se hace evidente tal situación. La empresa tabacalera Phillip Morris hace responsable al gobierno uruguayo por las violaciones cometidas a través de las políticas de control sobre el tabaco; demanda que es interpuesta ante el "Centro Internacional de Arreglos de Diferencia Relativas a Inversiones" (CIADI), del Banco Mundial. Este arguye que la legislación sobre el control del tabaco en Uruguay prohíbe el consumo en lugares públicos o cerrados (incluyendo los lugares de trabajo) y prohíbe la visibilidad de los cigarrillos en los centros comerciales (incluyendo uno de los avisos gráficos más fuertes en el mundo establecidos por la Organización Mundial de la Salud en la prevención sobre el consumo de tabaco, etc.). También, ha establecido de forma especial en dicha demanda las compensaciones a que haya lugar por las pérdidas ocasionadas a sus negocios, más la clausura de algunas normas; advirtiendo que Uruguay violó principalmente las políticas sobre protección de inversiones que firmó con Suiza, donde tiene su casa matriz la Phillip Morris.

Aunque el Sistema Interamericano de Derechos Humanos (SIDH) ha protegido los derechos a la propiedad comunitaria y al medio ambiente de los pueblos y comunidades, esto ocurre de manera lenta y costosa, puesto que acceder a este sistema no es sencillo, lo que conlleva que se necesiten asesorías en técnica jurídica y a que en tanto se tramitan los procesos legales, los pueblos y comunidades sean explotados por las grandes corporaciones mientras se decretan las medidas provisionales o se expide el fallo respectivo. A modo de ejemplo:

- El caso de los pobladores de la Comunidad Mayagna (Sumo) Awas Tingni Vs Nicaragua donde se buscaba proteger a la comunidad de la explotación maderera y actividades agropecuarias en 62 mil ha concedidas por el Estado a la empresa privada Sol del Caribe, sin que se hiciera una consulta previa a la comunidad. El caso fue presentado a la Corte Interamericana el 2 de octubre de 1995 y solo fue fallado hasta el 31 de agosto de 2001.

- Por otro lado, está el caso de las Cuatro Comunidades Indígenas Ngobe y sus miembros Vs Panamá; se otorga a la empresa privada AES Changuinola la administración de 6.215 ha de bosque y la posibilidad de construir represas, sin realizar la consulta previa a las comunidades, además de que suponía la inundación del territorio donde se encontraban más de 3.000 personas pertenecientes a las distintas comunidades. Las medidas provisionales se solicitan el 19 de enero de 2010 y se niegan el 28 de mayo de 2010, porque no se demuestra la extrema gravedad y urgencia.

- En el caso del pueblo indígena Kichwa de Sarayaku Vs Ecuador se otorgaron concesiones de explotación minera en 200.000 ha de tierra, generando amenazas contra la comunidad, deterioro de fuentes de agua, destrucción de bosques, cuevas, lugares sagrados y desplazamiento de la fauna. La solicitud se presenta el 26 de abril de 2010 y se falla el 27 de junio de 2012. 
- Para el caso de las Comunidades del Jiguamiandó y del Curbaradó vs Colombia se buscaba la protección de las comunidades del Chocó frente a la siembra de palma africana y la explotación de los recursos naturales que promovía una empresa privada (URAPALMA) desde el 2001 en 1500 ha y que suponía el peligro a la vida y supervivencia de las comunidades, puesto que se ocasionaron desapariciones forzadas, desplazamientos y amenazas contra los pobladores. En el 2003 la Corte Interamericana decreta medidas provisionales que garanticen la protección de los pobladores y se levantan en el 2013.

Por todo lo anterior, es necesario que en el tema de bioeconomía se tengan políticas claras y precisas por parte del Estado colombiano, en cuanto a los procesos regulatorios para desarollar y/o innovar en biotecnología agrícola usando los recursos genéticos, y sobre todo que se haga seguimiento al cumplimiento de las mismas para que no se vean afectadas de forma negativa las comunidades de las regiones y los investigadores como por ejemplo con el contrato de acceso a recursos genéticos (carg) el cual no ha terminado de legislarse completamente (Portafolio (2013).

\section{Tribunales ético-políticos de los pueblos}

A diferencia de los tribunales internacionales estructurados por el poder corporativo global que mantienen prácticas deplorables que menoscaban la vida comunitaria, destruyen el tejido social y las relaciones sacras entre éstas y la naturaleza, existen otros procesos alternativos. Tal es el caso de los tribunales éticos y políticos, que son ejercicios de los pueblos para pensar colectivamente el territorio y buscar justicia ante un Estado cómplice de las transnacionales, por lo que sus juicios éticos, morales y políticos permiten hacerle frente a la impunidad y salvaguardar todas las formas de vida, constituyéndose así en una respuesta a la lógica de las empresas que actúan de manera global; verbigracia el Tribunal Permanente de los Pueblos, un organismo internacional con sede en la fundación Lelio y Lisli Basso ISSOCO (Roma-Italia), que fue fundado en 1979 tras dos eventos, a saber, la adopción de la Declaración Universal de los Derechos de los Pueblos, en Argel en 1976 y la conclusión del segundo Tribunal Russell.

Junto al Tribunal Permanente de los Pueblos, se hallan otras experiencias como el Tribunal Latinoamericano del Agua (TLA), que es una instancia de justicia alternativa para el análisis y la búsqueda de solución a los crecientes conflictos hídricos. Su naturaleza éticojurídica y científico-técnica reformula el sentido del Derecho y actúa ante la crisis de legalidad respecto a las problemáticas relacionadas con el agua en Latinoamérica. (Tribunal Latinoamericano del Agua, s. f.)

En Colombia, después de una exigencia jurídica sobre las agresiones cometidas por las transnacionales a los derechos humanos, emana una experiencia del Colectivo de Abogados "José Alvear Restrepo" (2016); dado que trabajaron arduamente en los procesos de formación para lograr "facilitadores jurídicos en las regiones que defienden la población y protegen el territorio de las empresas transnacionales". Esto incluye una documentación de casos concretos, la facilidad en el uso normativo debido al acompañamiento y seguimiento de 
los procesos por parte del colectivo y otras organizaciones nacionales como es el caso de Censat agua viva- Amigos de la tierra Colombia (s. f.).

Otro proceso de Justicia Popular se dio en Argentina, con el juicio ético a las transnacionales que articulaba tres audiencias previas contra transnacionales extractivas: Trelew del 16 y 17 de septiembre del 2011; Audiencia del Juicio Ético en Patagonia; San Miguel de Tucumán del 23 al 25 de septiembre del 2011; y la Audiencia del Juicio Ético en el Noroeste Argentino (NOA); Wanda del 30 de septiembre y 1 de octubre del 2011 - Audiencia del Juicio Ético en la Triple Frontera (Argentina, Brasil y Paraguay), donde en sentencia final del Tribunal del Juicio Ético a las transnacionales. Buenos Aires, Compromiso del Tribunal Ético Popular declaraba:

Al concluir la audiencia de este Juicio Ético Popular a las Transnacionales, las y los participantes, integrantes de movimientos populares, de organizaciones de derechos humanos, investigadores/as, nos declaramos en estado de movilización, de unidad, de encuentro, de solidaridad, para borrar las fronteras entre nuestras resistencias a las políticas de recolonización del continente, para levantar una voz unánime en Nuestra América en defensa de la soberanía alimentaria, la agricultura familiar, el respeto a nuestras identidades y formas de organización y de vida, de nuestros derechos como hombres y mujeres, los derechos de las 183 generaciones venideras, los derechos de la naturaleza (Movimientos Sociales y Latinoamericanos. Centro de Investigación y Formación, s. f.).

La repercusión de estos casos es nacional e internacional y fortalece a las comunidades amenazadas por la imperiosa necesidad del capital extractivo por privatizar la vida y la biodiversidad; acabando con el tejido social, la economía responsable de las comunidades, la cultura, la soberanía alimentaria y el acceso a la justicia.

\section{Consideraciones finales}

La asimetría jurídica establecida por organismos multilaterales como el BM, FMI, OMC, OCDE, ONU, la SIDH -en algunos casos-, y los Estados de rectoría configuran una arquitectura jurídica de impunidad en las transacciones que se resuelve en beneficio de las multinacionales.

En vista de que los pueblos y las comunidades han identificado la asimetría de esta arquitectura acuden a instancias como los Tribunales ético-políticos y los Tribunales morales, cuyas sentencias buscan responsabilizar moral y financieramente a los reales causantes de los perjuicios englobando los impactos causados por sus intervenciones en los territorios.

En el tema tratado en este artículo, para hacer frente a la agenda política de la OCDE, los pueblos y las comunidades deben fortalecer sus procesos organizativos y las instancias alternativas de justicia como los tribunales éticos, políticos y morales que permitan ir del juicio ético, político y moral a una sanción real y efectiva. 
Es necesario propender por una bioeconomía orientada hacia la agricultura sostenible, en la que se tengan en cuenta las necesidades de las comunidades y una activa participación de éstas y de los investigadores conscientes de que es posible lograr un equilibrio entre estos factores.

\section{Conflicto de intereses}

Los autores declaran no tener ningún conflicto de intereses.

\section{Referencias bibliográficas}

1. Alto Comisionado de las Naciones Unidas para los Derechos Humanos. (2016). Consejo de Derechos Humanos. 31ํㅜ período de sesiones. Tema 2 de la agenda. Informe anual del Alto Comisionado de las Naciones Unidas para los Derechos Humanos e informes de la Oficina del Alto Comisionado y del Secretario General. Adición. Situación de los derechos humanos en Colombia. 21 p. Disponible en: http://www.acnur.org/t3/ fileadmin/Documentos/BDL/2015/9944.pdf?view=1

2. Boron, Atilio. (2007). El terrorismo como política de Estado. En: La Gente. Radio La Primerísima. 91.5- 105.3. Disponible en: http://www.radiolaprimerisima.com/ noticias/22705/el-terrorismo-como-politica-de-estado-por-atilio-boron

3. Censat agua viva- Amigos de la tierra Colombia. (s. f.). Sobre nosotros. Disponible en: http://censat.org/es/sobre-nosotros

4. Centro Nacional de Memoria Histórica (s.f.). Masacres del conflicto armado. Disponible en: $\quad$ http://centrodememoriahistorica.gov.co/descargas/informes2013/bastaYa/ basesDatos/Masacres1980-2012.xls

5. Colectivo de Abogados “José Alvear Restrepo" (CAJAR) (2016). Cajar recibirá premio de derechos humanos en Estados Unidos. Disponible en: http://www.colectivodeabogados. org/?-Quienes-Somos259-

6. Comisión Interamericana de Derechos Humanos (2011). Segundo informe sobre la situación de las defensoras y los defensores de derechos humanos en las Américas. Disponible en: https://www.oas.org/es/cidh/defensores/docs/pdf/defensores2011.pdf

7. Dávalos, Pablo. (2011). La democracia disciplinaria. El proyecto posneoliberal para América Latina. Bogotá, Ediciones Desde Abajo.

8. Giraldo, R. \& Nieto, L. (2015). Tendencies of biotechnology, innovation and development in Colombia. Revista Luna Azul, 41, 348-364. Disponible en: http://lunazul.ucaldas. edu.co/index.php?option=content\&task=view\&id=1070

9. Human Rights Watch (2015) Informe Mundial 2015: Colombia. Eventos de 2014. Disponible en: https://www.hrw.org/es/world-report/2015/country-chapters/268137

10. Molano, Alfredo. (2016). Desde Canadá. En: El Espectador. Disponible en: http://www. elespectador.com/opinion/canada-0

11. Movimientos Sociales y Latinoamericanos. Centro de Investigación y Formación (s. f.). Sentencia del Tribunal del Juicio Ético a las Transnacionales. Disponible en http:// www.cifmsl.org/index.php?option=com_content\&task=view\&id=1375\&Itemid=39

12. OECD. (2009). The Bioeconomy to 2030. Designing a Policy Agenda. Main Findings and Policy Conclusions. OECD International Futures Project. Available in: http://www. oecd.org/futures/longtermtechnologicalsocietalchallenges/42837897.pdf 
13. Peace Brigades International-PBI (2016). Informe sobre los riesgos, agresiones y retos de las personas defensoras de derechos humanos. Boletin informativo (21). ISSN 19083489. Disponible en: https://issuu.com/pbicolombia/docs/160223_eu_esp_a_1

14. Portafolio (2013). La bioeconomía en el país. Disponible en: http://www.portafolio.co/ opinion/redaccion-portafolio/bioeconomia-pais-69488

15. Publimetro (2016). Defensoría denuncia amenazas periodistas y lídres sindicales. Disponible en: http://www.defensoria.gov.co/es/nube/enlosmedios/4827/ Defensor\%C3\% ADa-denuncia-amenazas-a-periodistas-y-1\%C3\% ADderessindicales.htm

16. Tribunal Latinoamericano del Agua (s. f.). Fundamentos Éticos y Jurídicos del Tribunal Latinoamericano del Agua. Disponible en: http://tragua.com/quienes-somos/ fundamentos-vision-mision-objetivos/

17. Tribunal Permanente de los Pueblos (s. f.). Sesión sobre la industria minera canadiense. Disponible en: http://www.tppcanada.org/a-propos-du-tpp/pourquoi-une-session-surlindustrie-miniere/?lang=es 